This is the accepted manusript of the article: Peter Varga , Erik Grafarend, Johannes Engels, Relation of different type Love-Shida numbers determined with the use of time-varying incremental gravitational potential.

Appeared in: PURE AND APPLIED GEOPHYSICS, First Online: 22 March 2017., pp. 1-6. (2017), ISSN: $0033-4553$

Publisher's version:

https://link.springer.com/article/10.1007\%2Fs00024-017-1532-z 


\title{
Relation of different type Love-Shida numbers determined with the use of time-varying incremental gravitational potential
}

Peter Varga ${ }^{(1)}$, Erik Grafarend ${ }^{(2)}$, Johannes Engels ${ }^{(2)}$

(1) Seismological Observatory, Institute of Geodesy and Geophisics, (2) Department of Geodesy and Geoinformatics,, Stuttgart University

\begin{abstract}
There are different equations to describe relations between different classes of Love-Shida numbers. In this study with the use of the time-varying gravitational potential an integral relation was obtained which connects tidal Love-Shida numbers $(h, l, k)$, load numbers ( $h$ ', $l$ ', $k^{\prime}$ ), potential free Love-Shida numbers generated by normal ( $h$ ”, $l$ ”, $k$ ”) and horizontal $(h, ', l,, k$,', ) stresses. The equations obtained in frame of present study is the only one which

- holds for every type of Love-Shida numbers,

- describes a relationship not between different, but the same type of Love-Shida numbers,

- does not follows from the sixth-order differential equation system of motion usually applied to calculate the Love-Shida numbers.
\end{abstract}

\section{Keywords}

Love-Shida numbers, load numbers, potential free Love-Shida numbers, inhomogeneous motion equation systems

\section{Introduction}

The improvement of accuracy of Earth tidal observations and increase the length of the monitoring period, especially in the case of gravitational tidal observations, recently allows high resolution separation of near-frequency waves and precise determination of their amplitudes (Ducarme, 2012; Calvo et al, 2014; Van Camp et al., 2016). At the same time interpretation of observation results requires a calibration accuracy at the $1 \%$ o level (Meurers et al., 2016). This is important for the interpretation of Earth tide data, because of relatively poorly known amplitude ratios. The amplitude ratios obtained from observations carried out with gravimeters are $\delta_{2}=1+h_{2}-3 / 2 k_{2}$, with tiltmeters $\gamma_{2}=1+k_{2}-h_{2}$, with strainmeters $S_{\phi \phi 2}=h_{2}-4 l_{2}$ and $S_{\lambda \lambda}=h_{2}-2 l_{2}$ and with dilatometers $f_{2}=2 h_{2}-6 l_{2}[1$ $\Lambda /(\Lambda+2 \mu)]$, where $\Lambda$ and $\mu$ are Lamé parameters and $\phi$ and $\lambda$ the geographical latitude and longitude. On the basis of theoretical model calculations it can be assumed that $k_{2} \sim 0.5 h_{2}$ and $l_{2} \sim 0.15 h_{2}$ and at the surface of the Earth $(r=a) \quad \Lambda(a) \sim \mu(a), \delta_{2}=1+1 / 4 h_{2}, \gamma_{2}=1-k_{2}$, $S_{\phi \phi 2}=1 / 3 h_{2}, S_{\lambda \lambda 2}=2 / 3 h_{2}, f_{2}=3 / 4 h_{2}$. This means that deviation from the absolutely rigid, inelastic Earth in case of gravity Earth tides is only 15\%. In case of tilt and strain observations along latitude and longitude this deviations are $30 \%, 20 \%$ and $40 \%$ respectively, while for dilatational observations it is $45 \%$. The free core nutation due to the dynamical effect of the liquid core is more pronounced in case of strain and dilatation measurements than for gravimetric and tilts observations: 


$$
\begin{aligned}
& \delta_{O 1^{-}} \delta_{K 1}=0.021 \quad(1.8 \%) \\
& \gamma_{O 1^{-}} \gamma_{K 1}=-0.041 \quad(5.8 \%) \\
& S_{\phi \phi O 1^{-}} S_{\phi \phi K l}=0.098 \quad(33.7 \%) \\
& S_{\lambda \lambda O 1^{-}} S_{\lambda \lambda K 1}=0.192 \quad(37.5 \%) \\
& f_{O 1^{-}} f_{K 1}=0.117 \quad(23.7 \%)
\end{aligned}
$$

The amplitude ratios derived from observations provide linear combinations of Love and Shida numbers but do not provide the numbers themselves. At the same time for study of many different geodetic and geophysical problems the numbers and not their combinations are needed. It may be therefore important to determine relationships between the LoveShida numbers. A similarly important task is to derive relations between the tidal and other types of Love-Shida numbers, the load and potential free load numbers.

For the study of aforementioned problems the time-varying gravitational potential was determined in case of deformation generated by tide, tidal, load and potential free load with the use of the Love and Shida numbers in case of a symmetric non-rotating isotropic elastic model Earth. The Love-Shida numbers are determined in this case by the radial profiles of elastic Lamé parameters and density. Due to the mass conservation in case of elastic deformation the incremental mass density was inferred from the divergence of the product of initial mass density and the vertical displacement field.

\section{Equation of Melchior (1950) for the ratio of Love numbers $h_{n}$ and $k_{n}$}

For ratio of Love numbers $k_{n}$ and $h_{n}$ Melchior (1950) obtained

$$
\frac{k_{n}}{h_{n}}=\frac{n+3}{(2 n+1) a} \cdot \frac{\int_{0}^{a} \rho(r) r^{n+2} d r}{\int_{0}^{a} \rho(r) r^{2} d r}
$$

These ratios for $n=2-10$ and $n=20$ are shown on Fig. 1 .

In case of $n=2$

$$
\frac{k_{2}}{h_{2}}=\frac{1}{a} \cdot \frac{\int_{0}^{a} \rho(r) r^{4} d r}{\int_{0}^{a} \rho(r) r^{2} d r}
$$

The r.h.s. of this equation is a ratio of two Stokes constants: the mass $(M)$ and polar moment of inertia $(C)$ of the Earth. Consequently the ratio of the two Love numbers can be determined without any hypothesis concerning the inner structure of the Earth. According to Jeffreys (1959)

$$
\int_{0}^{a} \rho(r) r^{4} d r=\frac{3}{2} M a^{2}\left(1-\frac{2}{5} \sqrt{1+\eta(a)}\right)
$$

where $\eta(r)$ is the Radau function and for $r=a$ it is $\eta(a)=\frac{5}{2 \alpha} \cdot \frac{\omega^{2} a}{g(a)}=0.572$ (Moritz, 1990). Consequently $\frac{k_{2}}{h_{2}}=0.493$. 


\section{Relationships between different types of Love-Shida numbers}

The Love-Shida numbers can be obtained with the use of the inhomogeneous motion equation system given by Takeuchi (1953), Molodensky (1953), Alterman et al (1959). The solution of this system must satisfy three boundary conditions on the surface of the Earth which concern the normal stress $(N(a))$, the tangential stress $(M(a))$ and the derivative of the mass potential $(L(a))$ (Saito, 1978):

$$
\begin{aligned}
& N(a)=-\frac{2 n+1}{3} \cdot \alpha_{N} \\
& M(a)=\frac{2 n+1}{3 \cdot n \cdot(n+1)} \cdot \alpha_{M} \\
& L(a)=(2 n+1) \cdot \alpha_{L}
\end{aligned}
$$

where $\alpha_{N}, \alpha_{M}, \alpha_{L}$ can take the value 0 or 1 and $n$ is the order of spherical harmonic describing the deformation.

In case of Earth tides boundary conditions $\alpha_{N}=\alpha_{M}=0$ and $\alpha_{L}=1$ determine the LoveShida numbers $h_{n}, k_{n}$ and $l_{n}$. In case of a normal load acting on the surface of the Earth the triplet of parameters $\alpha_{N}=1 \alpha_{M}=0$ and $\alpha_{L}=1$ allows to determine the load Love-Shida numbers $h_{n}^{\prime}, k_{n}^{\prime}$ and $l_{n}^{\prime}$. If a potential free stress acting on the Earth surface $\left(\alpha_{L}=0\right)$ potential free Love-Shida numbers can be introduced (Molodensky, 1977) for normal $\alpha_{N}=1 \alpha_{M}=0 \alpha_{L}=0$ and tangential $\alpha_{N}=0 \alpha_{M}=1 \alpha_{L}=0$ stresses. The corresponding

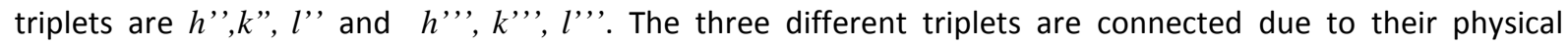
meaning. The load numbers are sums of deformation due to normal stress and gravitational effect of loading masses:

$$
\begin{aligned}
& h_{n}^{\prime}=h_{n}-\frac{2 n+1}{3} \cdot h_{n}^{\prime \prime} \\
& k_{n}^{\prime}=k_{n}-\frac{2 n+1}{3} \cdot\left(1+k_{n}^{\prime \prime}\right) \\
& l_{n}^{\prime}=l_{n}-\frac{2 n+1}{3} \cdot l_{n}^{\prime \prime}
\end{aligned}
$$

Furthermore according Molodensky (1977) the following equations can be introduced

$$
\begin{aligned}
& h_{n}^{\prime \prime \prime}=n(n+1) \cdot l_{n}^{\prime \prime \prime} \\
& h_{n}=\frac{2 n+1}{3} \cdot\left(1+k_{n}^{\prime \prime \prime}\right) \\
& l_{n}=\frac{2 n+1}{3 n(n+1)} \cdot\left(1+k_{n}^{\prime \prime \prime}\right)
\end{aligned}
$$

From (5b) it follows

$$
k_{n}^{\prime}=k_{n}-h_{n}
$$

The validity of (6) can be checked with computation of Love numbers $h_{n}, k_{n}$ and load number $k_{n}^{\prime}$ (Table 1). A comparison of $k_{n}^{\prime}$ and $k_{n}-h_{n}$ where the Love-Shida numbers were obtained with the use of boundary conditions $\alpha_{N}=\alpha_{M}=0 ; \alpha_{L}=1$ and the load number 
$k_{n}^{\prime}$ with $\alpha_{N}=1 \alpha_{M}=0$ and $\alpha_{L}=1$ allows to conclude that the calculations carried out with the differential equation system of Molodensky (1953) have deviation $\leq \pm 2 \cdot 10^{-4}$.

Table 1. Love numbers $h_{n}, k_{n}$ and load number $k_{n}^{\prime}$ calculated with the use of differential equation system of Molodensky (1953) on the basis of PREM for the degrees $n=2-4$.

\begin{tabular}{|c|c|c|c|c|}
\hline$n$ & $k_{n}$ & $h_{n}$ & $k_{n}^{\prime}=k_{n}-h_{n}$ & $k_{n}^{\prime}$ \\
\hline 2 & 0,3035 & 0,6176 & $-0,3141$ & $-0,3145$ \\
\hline 3 & 0,0944 & 0,2957 & $-0,2013$ & $-0,2012$ \\
\hline 4 & 0,0425 & 0,1781 & $-0,1356$ & $-0,1354$ \\
\hline
\end{tabular}

The gravitational potential of a deformable body generated by tidal, load and potential free potentials.

The aim of the next part of present study is to derive a mathematical expression which is composed by all three components of triplets and which is equally applicable for Love-Shida, for load and for potential free Love numbers. For this purpose an approach was developed which is not based on an inhomogeneous motion equation system given by Takeuchi (1953), Molodensky (1953), Alterman et al (1959).

The Newtonian equation for the time dependent deformation potential is (Grafarend et al., 1997)

$$
\Delta \omega\left(x_{a}, \mathrm{t}\right)=-G \iiint_{r \lambda \phi} \frac{\operatorname{div} \rho(x) d(x, t)}{\left\|x_{a}-x\right\|} d r d \lambda d \phi
$$

where $x$ is a source point within the Earth, $x_{a}$ is the field point on the surface $(r=a), \rho(x)$ and $d(x, t)$ characterize the initial mass density and the time dependent displacement vector of the deformable Earth. In (7) the continuity equation has been used

$$
\Delta \varrho(x, t)=-\operatorname{div}[\rho(x) d(x, t)]
$$

what means that there is no loss of mass during the deformation. Furthermore

$$
\Delta \varrho(x, t)=-\operatorname{div}[\rho(x) d(x, t)\rceil=-\varrho(x) \operatorname{div} d(x, t)+\frac{\operatorname{grad} \varrho(x)}{d(x, t)}
$$

The denominator in (7) could be expanded into the scalar surface spherical surface functions

$$
\frac{1}{\left\|x_{a}-x\right\|}=\frac{1}{a} \sum_{n=0}^{\infty} \sum_{m=-n}^{n} \frac{1}{2 n+1}\left(\frac{r}{a}\right)^{n} Y_{n, m}\left(\lambda_{a}, \phi_{a}\right) Y_{n, m}(\lambda, \phi)
$$

In the most general case the displacement vector expressed by orthonormal vector surface spherical functions is 


$$
d(x, t)=\sum_{n=0}^{\infty} \sum_{m=-n}^{n}\left[r^{n m}(r, t) R_{n m}(\lambda, \phi)+s^{n m}(r, t) S_{n m}(\lambda, \phi)+t^{n m}(r, t) T_{n m}((\lambda, \phi)]\right.
$$

On the r.h.s. of (9) the first two terms represent the spheroidal displacements. The third term stands for the toroidal displacement field $s$ connected to phenomena not discussed in this study. Furthermore with unit vectors $e_{r}, e_{\lambda}, e_{\phi}$ one can write

$$
\begin{aligned}
& R_{n m}=e_{r} Y_{n, m}(\lambda, \phi) \\
& S_{n m}=\frac{1}{\sqrt{n(n+1)}}\left(\frac{e_{\lambda}}{\cos \phi} \frac{\partial}{\partial \lambda} Y_{n, m}(\lambda, \phi)+e_{\phi} \frac{\partial}{\partial \phi} Y_{n, m}(\lambda, \phi)\right)
\end{aligned}
$$

With the use of equations (7)-(10) the time dependent deformation potential is

$$
\begin{gathered}
\Delta \omega\left(x_{a}, t\right)=-\frac{G}{a} \iiint_{r \lambda \phi} \sum_{n=0}^{\infty} \sum_{m=-n}^{n} \frac{1}{2 n+1}\left(\frac{r}{a}\right) Y_{n m}\left(\lambda_{a}, \phi_{a}\right) \cdot Y_{n m}(\lambda, \phi) \cdot \\
\cdot \sum_{i=0}^{\infty} \sum_{j=-i}^{i}\left\{\left[\frac{2}{r} r^{i j}+\frac{d}{d r} r^{i j}-\frac{\sqrt{i(i+1)}}{r} s^{i j}\right] \cdot \rho(x) Y_{i j}(\lambda, \phi)+\frac{1}{r \cos \phi} \cdot \frac{\partial \rho}{\partial \lambda} .\right. \\
\left.\cdot\left[\frac{s^{i j}}{\sqrt{i(i+1)}} \frac{1}{\cos \phi} \frac{\partial}{\partial \lambda} Y_{i j}(\lambda, \phi)\right]+\frac{1}{r} \frac{\partial \varrho}{\partial \phi} \mid \frac{s^{i j}}{\sqrt{i(i+1)}} \frac{\partial}{\partial \phi} Y_{i j}(\lambda, \phi)\right] \\
\left.+\frac{\partial \varrho}{\partial r}\left[r^{i j} Y_{i j}(\lambda, \phi)\right]\right\} d r d \lambda d \phi
\end{gathered}
$$

The tidal displacement components could be represented as

$$
\begin{gathered}
d_{\lambda}=l(r)(\gamma r \cos \phi)^{-1} \frac{\partial V_{T}}{\partial \lambda} \\
d_{\phi}=l(r)(\gamma r)^{-1} \frac{\partial V_{T}}{\partial \phi} \\
d_{r}=h(r) \gamma^{-1} V_{T}
\end{gathered}
$$

where $\gamma$ is the mean gravity acceleration and the tidal potential expressed with spherical surface function is

$$
V_{T}=\sum_{n=0}^{\infty} \sum_{m=-l}^{l} V_{n m} Y_{n m}\left(\lambda_{a}, \phi_{a}\right)
$$

Consequently $r^{n m}(r)=h_{n}(r) \gamma^{-1} V_{n m}$ and $s^{n m}(r)=l_{n}(r) \gamma^{-1} \sqrt{n(n+1)} V_{n m}$

Due to the radial symmetry of the Earth model used in this paper $\partial \varrho / \partial \lambda=\partial \varrho / \partial \phi=$ $0, \partial h / \partial \lambda=\partial h / \partial \phi=0$ and $\partial l / \partial \lambda=\partial l / \partial \phi=0$. Consequently (11) can be given in a simpler form: 


$$
\begin{aligned}
& \Delta \omega_{n m}(a, t)=-G \iiint_{r \lambda \phi}\left\{[ \rho ( r ) ( \frac { 1 } { a } ) \cdot \frac { 1 } { 2 n + 1 } ( \frac { r } { a } ) ^ { n } Y _ { n m } ( \lambda _ { a } , \phi _ { a } ) Y _ { n m } ( \lambda , \phi ) ] \cdot \left\{\left[\frac{2}{\gamma r} h_{n}(r)+\left(\frac{1}{\gamma}\right) \frac{\partial h_{n}(r)}{\partial r}-\right.\right.\right. \\
& \left.\left.\left.\frac{i(i+1)}{\gamma r} l_{n}(r)\right] V_{i j} Y_{i j}(\lambda, \phi)+\left[\left(\frac{1}{\gamma}\right) \frac{\partial \varrho(r)}{\partial r} h_{n}(r)\right]^{i} V_{i j} Y_{i j}(\lambda, \phi)\right\}\right\}
\end{aligned}
$$

With the use of orthonormality relations of the surface spherical harmonics (12) can be given in form:

$\Delta \omega_{n m}(a, t)=-4 \pi G \int_{0}^{a}\left\{\frac{\varrho(r)}{a}\left[\frac{1}{2 n+1} Y_{n m}\left(\lambda_{a}, \phi_{a}\right)\right] \cdot\left[\frac{2}{\gamma r} h_{n}(r)+\frac{1}{r} \frac{d h_{n}(r)}{d r}-\frac{n(n+1)}{\gamma r} l_{n}(r)\right] V_{n m}+\right.$ $\left.\left[\frac{d \varrho(r)}{d r} \cdot \frac{1}{\gamma} h_{n} V_{n m}\right]\right\} \cdot\left(\frac{r}{a}\right)^{n} r^{2} d r=-\frac{4 \pi G Y_{n m}\left(\lambda_{a}, \phi_{a}\right) V_{n m}}{\gamma a(2 n+1)} \int_{0}^{a}\left\{\rho(r)\left[2 \frac{h_{n}(r)}{r}+\frac{d h_{n}(r)}{d r}-\right.\right.$ $\left.\left.\frac{n(n+1) l_{n}(r)}{r}\right]+h_{n}(r) \frac{d \varrho(r)}{d r}\right\}\left(\frac{r}{a}\right)^{n} r^{2} d r$

Using some simplifications (13) can be written as

$\Delta \omega_{n m}(a, t)=-4 \pi G /\left[\gamma(2 n+1) a^{n+1}\right] V_{n m} Y_{n m}\left(\lambda_{a}, \phi_{a}\right) \cdot \int_{0}^{a}\left\{\rho(r)\left[\frac{d h_{n}(r)}{d r} r^{n+2}+\right.\right.$ $\left.\left.2 h_{n}(r) r^{n+1}-n(n+1) l_{n}(r) r^{n+1}\right]+\frac{d \varrho(r)}{d r} h_{n}(r) \cdot r^{n+2}\right\} d r$

The partial integration of the last term of (14) gives

$$
\int_{0}^{a} \frac{d \varrho(r)}{d r} h_{n}(r) \cdot r^{n+2}=-\int_{0}^{a} \varrho(r)\left[\frac{d h_{n}(r)}{d r} r^{n+2}+h_{n}(r)(n+2) r^{n+1}\right] d r
$$

Therefore (14) may be simplified:

$\Delta \omega_{n m}(a, t)=4 \pi G /\left[\gamma(2 n+1) a^{n+1}\right] V_{n m} Y_{n m}\left(\lambda_{a}, \phi_{a}\right) \cdot \int_{0}^{a} \rho(r)\left[n(n+1) l_{n}(r) r^{n+1}+\right.$ $\left.n h_{n}(r) r^{n+1}\right] d r$

The r.h.s. of (15) gives the potential variation generated by deformation of the Earth which can be written as

$$
\Delta \omega_{n m}(a, t)=k_{n}(a) V_{n m} Y_{n m}\left(\lambda_{a}, \phi_{a}\right)
$$

where $k_{n}(a)$ is the Love number to describe the potential variations due to deformations. Connection of (15) and (16) leads to a relation between the Love-Shida numbers:

$$
k_{n}(a)=4 \pi G n /\left[\gamma(2 n+1) a^{n+1}\right] \cdot \int_{0}^{a}-\rho(r) r^{n+1}\left[n(n+1) l_{n}(r)+h_{n}(r)\right] d r
$$

With the use of relative system of units: the unit of distance is $a$, the unit of the gravity the mean gravity acceleration $\gamma$ and the unit of the density is the mean density of the Earth $\left(\rho_{\text {mean }}=5514 \mathrm{~kg} / \mathrm{m}^{3}\right)$. In this system $G=3 / 4 \pi$, and (17) will be

$$
k_{n}(a)=\frac{3}{2 n+1} \int_{0}^{a} \varrho(r) \cdot r^{n+1}\left[(n+1) l_{n}(r)+h_{n}(r)\right]
$$

And consequently for $n=2,3,4$

$$
\begin{gathered}
k_{2}(a)=\frac{3}{5} \int_{0}^{a} \varrho(r) \cdot r^{3}\left[3 l_{n}(r)+h_{n}(r)\right] \\
k_{3}(a)=\frac{3}{7} \int_{0}^{a} \varrho(r) \cdot r^{4}\left[4 l_{n}(r)+h_{n}(r)\right] \\
k_{4}(a)=\frac{3}{9} \int_{0}^{a} \varrho(r) \cdot r^{5}\left[5 l_{n}(r)+h_{n}(r)\right]
\end{gathered}
$$

\section{Conclusions}


The importance of equations (18) - (21) is that they are only relations which do not follow from the differential equation of motion usually used to calculate the Love-Shida numbers and consist the complete triplet $h_{n}, k_{n}$ and $l_{n}$ of the same type. They were obtained without any considerations concerning the boundary conditions ( 3 ) and therefore they are valid both for tidal, load and potential free Love-Shida numbers.

With the use of (19) $k_{2}(a)=0.3031$ which is close to value in Table 1 (0.3035).

On the r.h.s. of equations (18) - (21) the first term describes the horizontal and the second the vertical displacements. Their contribution to the value of Love number $k$ is almost equal to 0.148 and 0.146 respectively in the case of $n=2$. In case of the load numbers this ratio is different and significantly decreases with increasing $n: 0,589$ in case of $n=2$ and 0.097 for $n=10$.

\section{Acknowledgement}

We thank the Guest Editor David Crossley and an anonymous reviewer colleague for their helpful comments.

The research described in this paper was completed during research stay of P. Varga (01.03.2016-31.05.2016) supported by the Alexander Humboldt Foundation at the Department of Geodesy and Geoinformatics, Stuttgart University. P. Varga thanks Professor Nico Sneeuw for the excellent research conditions provided by him.

Financial support from the Hungarian Scientific Research Found OTKA (Project K125008) is acknowledged.

\section{References}

Alterman, Z., Jarosch, H.,\& Pekeris, C.L. (1959) Oscillations of the Earth, Proceedings of the Royal Society London A, 252, 80-95.

Calvo, M., Hinderer, J., Rosat, S., Legros, H., Boy, J.-P., Ducarme, B.,\& Zürn, W. ( 2014)

Time stability of spring and superconducting gravimeters ", through the analysis of very long gravity records, Journal of Geodynamics

Ducarme, B., 2012. Determination of the main lunar waves generated by the third degree tidal potential and validity of the corresponding body tides models, Journal of Geodesy, 86, 1, 65-75.

Grafarend, E., Engels, J., \& Varga, P. ( 1997) The spacetime gravitational field of a deformable body. Journal of Geodesy, 72, II-30.

Jeffreys H. (1959) The Earth its origin, history and physical constitution, Cambridge, University Press

Melchior, P.J. (1950) Sur l'influence de la loi de répartition des densités á l'intérieur de la Terre dans les variations Luni-Solaires de lagravité en un point, Geophysica Pura et Applicata, 16, 3-4, 105-112. 
Meurers., B., Van Camp M., Francis O.,\& Pálinkáš V. (2016) Temporal variation of tidal parameters in superconducting gravimeter time-series, Geophysical Journal International, 205, 1, 284-300.

Molodensky, M. S. (1953) Elastic tides, free nutations and some questions concerning the inner structure of the Earth, Trudi Geofizitseskogo Instituta Akademii Nauk of the USSR, , 19 (146), 3-42.

Molodensky, S.M. (1977) On the relation between the Love numbers and the load coefficients, Fizika Zemli, 3, 3-7.

Moritz, H. (1990) The figure of the Earth: theoretical geodesy and the Earth's interior, Wichmann, Karlsruhe.

Takeuchi, H. (1953) On the Earth tide of the compressible Earth of variable density and elasticity, Transactions American Geophysical Union, 31 (5), 651-689.

Saito, M. (1978) Relationship between tidal and load numbers, Journal of Physics of the Earth, 26, 13-16

Van Camp, M., Meurers, B., de Viron, O. \& Forbriger, Th. (2016) Optimized strategy for the calibration of superconducting gravimeters at the one per mille level, Journal of Geodesy., 90, 1, 91-99

Figure 1. Ratios $k_{n} / h_{n}$ for $n=2-10$ and 20

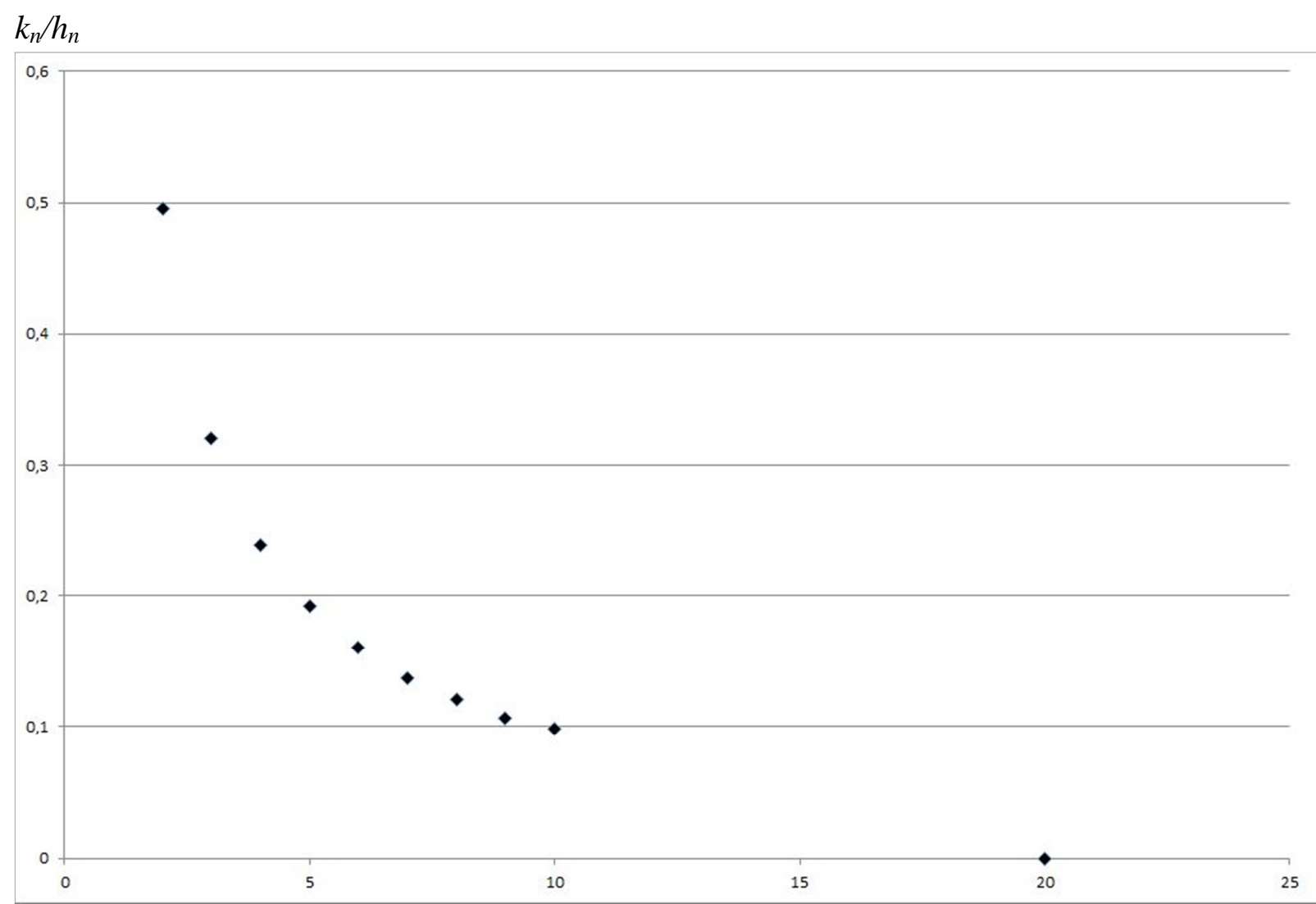


